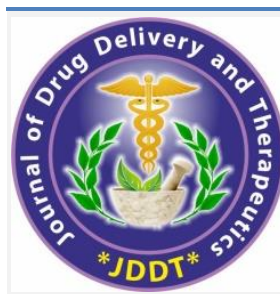

Open

Access Full Text Article
Available online on 15.03.2021 at http://jddtonline.info

\section{Journal of Drug Delivery and Therapeutics}

Open Access to Pharmaceutical and Medical Research

(C) 2011-21, publisher and licensee JDDT, This is an Open Access article which permits unrestricted non-commercial use(CC By-NC), provided the original work is properly cited

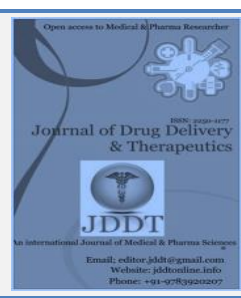

Research Article

\title{
Mesenteric lymphadenitis; a common diagnostic mimic to acute appendicitis. With radiology it is no more a diagnosis of exclusion, laparoscopy or surgery
}

\author{
Kamel El-Reshaid *, Shaikha Al-Bader **, Zhaneta Markova*** \\ * Department of Medicine, Faculty of Medicine, Kuwait University \\ ** Department of Medicine, kidney unit, Al-Amiri Hospital, Kuwait \\ *** Department of radiology, Qutaina medical center, Kuwait
}

\section{Article Info:}

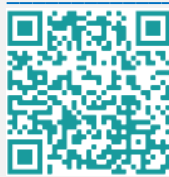

\section{Article History:}

Received 18 Jan 2021; Review Completed 20 Feb 2021 Accepted 27 Feb 2021; Available online 15 March 2021

\section{Cite this article as:}

El-Reshaid K, Al-Bader S, Markova Z, Mesenteric lymphadenitis; a common diagnostic mimic to acute appendicitis. With radiology it is no more a diagnosis of exclusion, laparoscopy or surgery,

Journal of Drug Delivery and Therapeutics. 2021; 11(2):55-57

DOI: http://dx.doi.org/10.22270/jddt.v11i2.4590

\section{Abstract}

Mesenteric lymphadenitis (ML) is an inflammatory response to viral infections, bacterial infections, inflammatory bowel disease, or lymphoma in the ileocecal regions. It is a common diagnostic mimic to acute appendicitis. It is a self-limited inflammatory process that affects mesenteric lymph nodes in the right lower quadrant (RLQ). We present a 15 year-old-girl with low grade fever and RLQ pain. Initial physical examination and laboratory investigations suggested acute appendicitis. However, diagnosis of ML was established by the finding abnormal lymph nodes that are usually: (a) clusters of $>3$ tender ones anterior to right psoas muscle or in the small bowel mesentery, (b) $>5 \mathrm{~mm}$ in diameter of short axis, and (b) rounded in shape. In addition; the ileal or ileocecal wall is thickened ( $>3 \mathrm{~mm}$ ) over at least $5 \mathrm{~cm}$ of the bowel and the appendicular wall is normal and its lumen is patent. In conclusion; this case reports indicates that with radiology; ML can be differentiated from acute appendicitis and limits the role of invasive laparoscopy or surgery in exclusion of the latter.

Keywords: appendicitis, CT, mesenteric lymphadenitis, radiology, US

\section{*Address for Correspondence:}

Dr. Kamel El-Reshaid, Professor, Dept. Of Medicine, Faculty of Medicine, Kuwait University, P O Box 24923, 13110 Safat, Kuwait

\section{INTRODUCTION}

Mesenteric adenitis (ML) is a self-limited inflammatory process that affects mesenteric lymph nodes in the right lower quadrant and is clinically often thought initially to be an acute appendicitis, a common diagnostic mimic 1. The true incidence of ML is not known, since it can be easily missed or mistaken for other diagnoses. In 70 case series of suspected acute appendicitis; $16 \%$ had a final diagnosis of ML later via surgery, imaging, or clinical course ${ }^{2}$. In this case report; we highlight the role of radiology in its diagnosis in an attempt to limit the invasive approach of laparoscopy and/or surgery.

\section{THE CASE:}

A 15 years-old girl presented with watery diarrhea for 3 days followed by 2 days of low grade fever, right iliac pain and vomiting. Initial physical examination right lower quadrant abdominal tenderness. Laboratory investigations showed high peripheral leucocytic count with neutrophila with normal platelets count and hemoglobin. Serum sugar, renal and liver profiles were normal. Urine routine and microscopy were normal. Stool showed excess pus and blood cells. Ultrasound examination suggested lymphadenopathy in the right iliac region of the abdomen. Computed tomography (CT) scan of the abdomen confirmed the diagnosis (figure 1). Stool culture revealed Yersinia enterocolitica. Since, she had persistent diarrhea and abdominal pains; she was treated with Ciprofloxacin $250 \mathrm{mg}$ twice daily for 5 days and had recovered completely. 


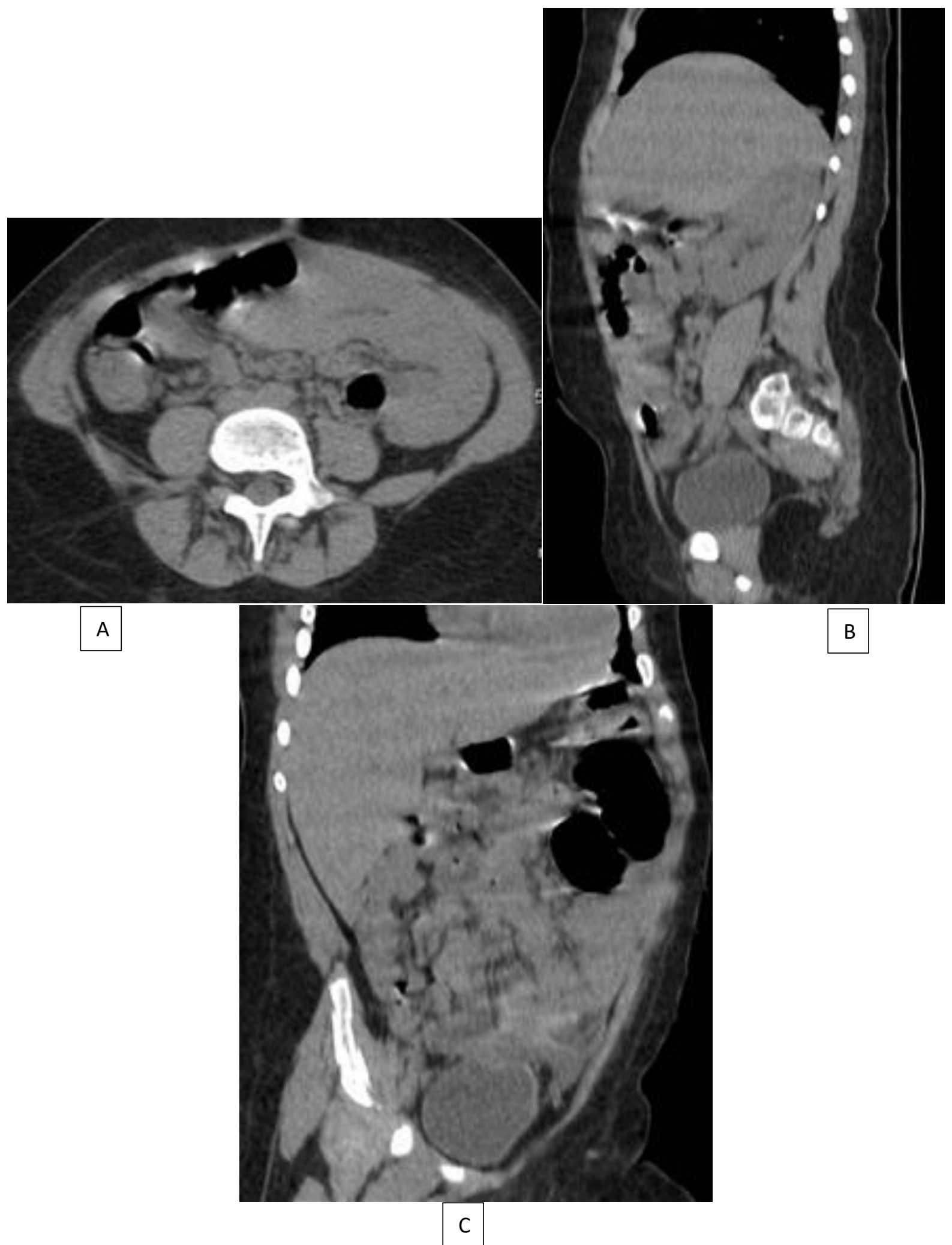

Figure 1. CT scan showing enlarged and rounded-shaped mesenteric lymph nodes without fat stranding in axial (A), sagittal (B) and coronal (c) views.

\section{DISCUSSION}

ML is an inflammatory response to viral infections, bacterial infections, inflammatory bowel disease, or lymphoma in the ileocecal regions. Yersinia enterocolitica is considered the most common pathogen in temperate Europe, North America and Australia though the disease has been reported with Helicobacter jejuni, Campylobacter jejuni, Salmonella species, Shigella species, and Mycobacterium tuberculosis.
Secondary ML can be associated with inflammatory conditions viz. Crohn's disease, SLE and diverticulitis as well as colonic malignancy. Immunocompromized patients may have HIV, lymphoma, Mycobacterium avium complex, Tuberculosis, Cryptosporium and even Kaposi's sarcoma 3. In the infectious category; the pathogenic micro-organisms are thought to gain access via intestinal lymphatics and then multiply in mesenteric lymph nodes. On gross pathology, lymph nodes are enlarged and soft. On microscopy, there is 
non-specific hyperplasia and when suppurative, there is necrosis and pus. Diagnosis is established by finding abnormal lymph nodes that are usually: (a) clusters of $>3$ tender ones anterior to right psoas muscle or in the small bowel mesentery, (b) $>5 \mathrm{~mm}$ in diameter of short axis, and (b) rounded in shape. In addition; the ileal or ileocecal wall is thickened ( $>3 \mathrm{~mm}$ ) over at least $5 \mathrm{~cm}$ of the bowel and the appendicular wall is normal and its lumen is patent 4 . The clinical Presentation and laboratory investigations of ML are similar to acute appendicitis. Diagnosis used to be of exclusion or at surgery or laparoscopy yet with ultrasonography and multidetector CT scan pathological ML can be detected and diagnosis is established. Moreover, radiological scans is useful to exclude secondary causes of ML as well as intussusception, ovarian cyst rupture, ovarian abscess, ectopic pregnancy, endometriosis, ovarian torsion, testicular torsion, epididymitis, pelvic inflammatory disease, mesenteric ischemia, chronic abdominal pain, inflammatory bowel diseases such as Crohn's or ulcerative colitis, systemic lupus erythematous, malignancy, HIV, zoonotic infections, infectious mononucleosis, and tuberculosis ${ }^{5}$. ML is usually a self-limited disease and complications are rare. However, in rare occasions, bacterial spread can lead to acute fulminant necrotizing lymphadenitis with abscess formation 6 Moreover, the enlarged mesenteric lymph nodes led to vascular compromise and ischemic colitis 7 . Contrary to acute appendicitis which requires urgent intervention to avoid rupture and sepsis; ML is a self-limited disease that usually require symptomatic treatment of fever and pain is with Acetaminophen or NSAIDs and fluids for dehydration from diarrhea. Given the predominance of Yersinia enterocolitica, initial antibiotic selection from
Trimethoprim-sulfamethoxazole, third-generation Cephalosporins, Fluoroquinolones, Aminoglycosides, and Doxycycline should be considered 8 . These agents provide broad coverage for enteric pathogens. In conclusion; ML ultrasonography and CT scan of the abdomen are essential in diagnosis of lesions right iliac fossa. With such high prevalence of ML and cost-effective approach; they can limit the invasive approach of laparoscopy and/or surgery in suspected cases of acute appendicitis.

\section{REFERENCES:}

1- Ian A. "Acute Non-Specific Mesenteric Lymphadenitis". Br Med J 1945; 2:680-682.

2- Vignault F, Filiatrault D, Brandt ML, Garel L, Grignon A, Ouimet A. Acute appendicitis in children: evaluation with US. Radiol. 1990; 176:501-504.

3- Helbling, R., Conficconi, E., Wyttenbach, M., Benetti, C., Simonetti, G. D., Bianchetti, M. G., Hamitaga, F., Lava, S. A., Fossali, E. F., \& Milani, G. P. (2017). Acute Nonspecific Mesenteric Lymphadenitis: More Than "No Need for Surgery". BioMed res int. 2017, 9784565.

4- Lucey BC, Stuhlfaut JW, Soto JA. Mesenteric lymph nodes: detection and significance on MDCT. AJR Am J Roentgenol. 2005; 184:41-44.

5- Sahai S. Lymphadenopathy. Pediatr Rev. 2013; 34: 216-227.

6- Carr JA. Acute fulminant necrotizing mesenteric lymphadenitis causing bowel ischemia. J Surg Case Rep.2019; 2019 (11):rjz304. doi: 10.1093/jscr/rjz304.

7- Birkhold M, Langenburg S. Is mesenteric adenitis a benign condition? Ischemic colitis secondary to mesenteric adenitis in a 12 year old. J Pediatr Surg Case Rep. 2016; 15:19-21.

8- Zinczuk J, Wojskowicz P, Kisluk J, Fil D, Kemona A, Dadan J. Mesenteric lymphadenitis caused by Yersinia enterocolitica. Prz Gastroenterol. 2015; 10:118-21. 